\title{
SIMULTANEOUS MEASUREMENT OF 2 AND 3 SPINS IN PROTON PROTON ELASTIC SCATTERING AT $6 \mathrm{GeV} / c^{\text {औ }}$
}

\author{
R.C. FERNOW, S.W. GRAY, A.D. KRISCH, \\ H.E. MIETTINEN, J.B. ROBERTS, K.M. TERWILLIGER \\ Randall Laboratory of Physics, The University of Michigan, Ann Arbor, Michigan 48104, USA \\ W. DeBOER \\ The University of Michigan, USA \\ and CERN, Geneva 23, Switzerland \\ E.F. PARKER and L.G. RATNER \\ Accelerator Research Facilities Division, \\ Argonne National Laboratory, Argonne, Illinois 60439, USA \\ J.R. O'FALLON \\ Department of Physics, St. Louis University, St. Louis, Missouri 63103, USA
}

Received 22 August 1974

\begin{abstract}
The elastic cross section for proton proton scattering at $6 \mathrm{GeV} / c$ was measured using a $70 \%$ polarized beam and a $75 \%$ polarized target at the Argonne ZGS. In the range $P_{\perp}^{2}=0.5 \rightarrow 2.0(\mathrm{GeV} / c)^{2}$ we obtained small error measurements for the $\uparrow \uparrow, \downarrow \downarrow$ and $\uparrow \downarrow$ initial spin states perpendicular to the scattering plane. At $P_{\perp}^{2}=0.5$ we also measured the recoil spin and found that the 5 different cross sections were very unequal.
\end{abstract}

There has been an increasing interest in the spin dependence of high energy strong interactions. This started with the very successful experiments using polarized proton targets at Berkely [1], CERN [2] and Argonne [3]. The new ZGS polarized proton beam allows new and even more precise measurements of this spin dependence.

We recently constructed, in cooperation with Argonne, a ${ }^{3} \mathrm{He}$ cryostat polarized proton target (PPT V), which is a copy of a CERN target. This target is maintained at $0.5 \mathrm{~K}$ in a field of $25 \mathrm{kG}$ and contains propanediol dpoed with $\mathrm{K}_{2} \mathrm{Cr}_{2} \mathrm{O}_{7}$. The protons interact with the electrons in the $\mathrm{Cr}$ and are pumped into a polarized state by a $70 \mathrm{GHz}$ microwave carcinotron tube. The proton polarization was measured with a $107 \mathrm{MHz}$ NMR system using signal averaging to be $P_{\mathrm{T}}=75 \pm 5 \%$. The PPT II target used for our earlier

\footnotetext{
Supported by a research grant from the U.S. Atomic Energy
} Commission. runs $[4,5]$ gave about $35 \%$.

The extracted polarized beam intensity increased by a factor of 10 to an average of $3 \times 10^{8}$ per $2.5 \mathrm{sec}$ pulse. The internal ZGS intensity was as high as $1.3 \mathrm{X}$ $10^{9}$ per pulse and the average beam polarization was $P_{\mathrm{B}}=69 \pm 4 \%$ with a peak of $75 \%$. These increases allowed considerable improvement in the precision of our measurements of the spin dependence of p-p elastic scattering.

The experimental apparatus shown in fig. 1 is almost identical to that used in our earlier measurements $[4,5]$. The beam polarization $\left(P_{\mathrm{B}}\right)$ was obtained from the high energy polarimeter which simultaneously measures pp elastic scattering from a liquid hydrogen target to the left $\left(\mathrm{L}=\mathrm{L}_{1} \mathrm{~L}_{2} \mathrm{~L}_{3} \mathrm{~L}_{4} \mathrm{~L}_{5} \mathrm{~L}_{6}\right)$ and to the right $\left(R=R_{1} R_{2} R_{3} R_{4} R_{5} R_{6}\right)$. The beam polarization is given by

$$
P_{\mathrm{B}}=\frac{1}{A}\left(\frac{\mathrm{L}-\mathrm{R}}{\mathrm{L}+\mathrm{R}}\right)
$$




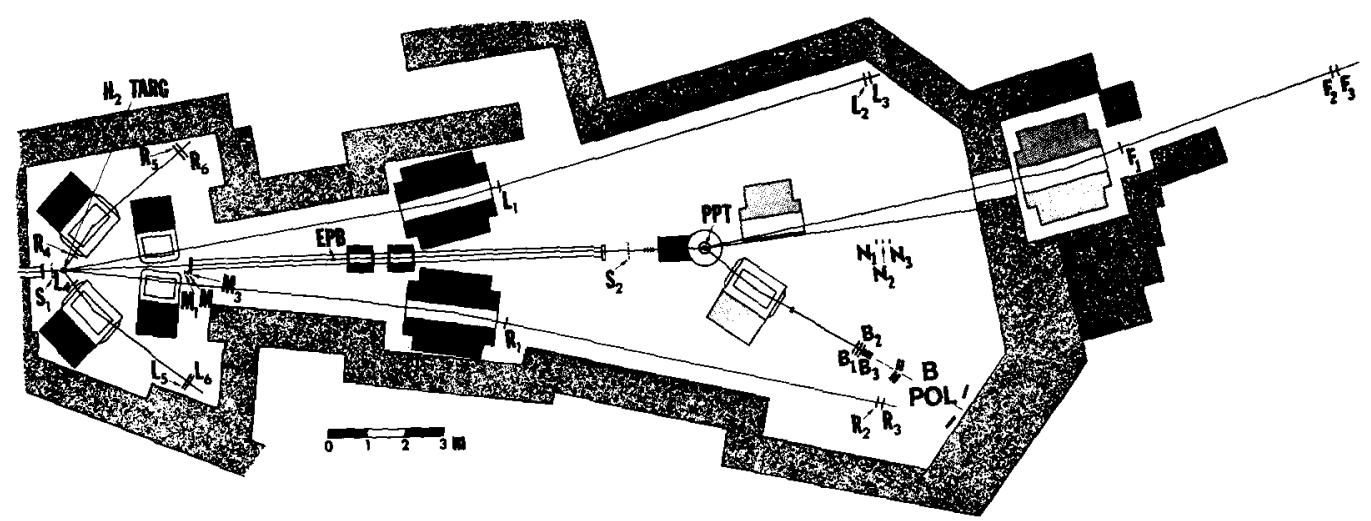

Fig. 1. Layout of the experiment. The polarized beam passes through the $\mathrm{H}_{2}$ target and its polarization is measured by comparing the number of elastic events seen in the $L$ and $R$ spectrometers of the polarimeter. The beam then scatters in the polarized proton target and the elastic events are counted by the $F$ and $B$ counters. The $\mathrm{M}$ and $\mathrm{N}$ counters are monitors. The B polarimeters measures the spin of the recoil proton by measuring p-Carbon scattering to the left and to the right.

where $A=0.100 \pm 0.006$ is the asymmetry parameter for p-p elastic scattering at $6 \mathrm{GeV} / c$ and $P_{\perp}^{2}=0.5$ $(\mathrm{GeV} / c)^{2}$ where the polarimeter was set.

The elastic cross section for the scattering of the polarized proton beam on the polarized proton target was measured using the downstream double arm spectrometer containing magnets and scintillation counters $\left(\mathrm{F}_{1} \mathrm{~F}_{2} \mathrm{~F}_{3} \mathrm{~B}_{1} \mathrm{~B}_{2} \mathrm{~B}_{3}\right)$. The solid angle of $\Delta \Omega_{1 \mathrm{ab}} \sim$ $5.7 \times 10^{-5}$ sr was defined by the $\mathrm{F}_{3}$ counter $6^{\prime \prime} \times 5^{\prime \prime}$ at $725^{\prime \prime}$ from the PPT. $F_{3}$ also defined the momentum bite of about $\triangle P / P= \pm 7 \%$. The $B$ counters which detect the recoil proton were now very overmatched since they were moved upstream to allow installation of the B-polarimeter. The F-B accidentals were monitored and subtracted and were normally less than $5 \%$. Magnet curves showed that inelastics were normally less than $2 \%$.

The quadrupoles installed between the two targets reduced the beam size at the PPT to about $13 \mathrm{~mm}$ FWHM. The beam was kept centered to about $2 \mathrm{~mm}$ using segmented wire ion chambers $S_{1}$ and $S_{2}$. This reduced systematic errors due to the beam moving in the $29 \mathrm{~mm}$ diameter target. Systematic errors were further reduced by flipping the direction of the beam spin every $1 \mathrm{hr}$ and the target spin every $8 \mathrm{hr}$, and were generally below $1 \%$.

The elastic cross sections was obtained in principle from

$$
\frac{\mathrm{d} \sigma}{\mathrm{d} \Omega}(i j)=\frac{\text { events }(i j)}{I_{\mathrm{o}} N_{\mathrm{o}} \rho t \Delta \Omega},
$$

where $I_{\mathrm{o}}$ is the number of incident particles, $N_{\mathrm{o}}$ is Avogadro's number, and $t$ is the target length of $3 \mathrm{~cm}$. The density $\rho$ of hydrogen protons in the PPT is about $0.07 \pm 0.015$. It was difficult to measure $I_{\mathrm{o}}$ absolutely, thus there is a normalization uncertainty in the absolute cross sections which is fortunately absent in the relative cross sections for the four different initial spin states $\uparrow \uparrow, \downarrow \downarrow, \uparrow \downarrow, \downarrow \uparrow$. We used the $N$ scintillation telescope to monitor the relative beam intensity through the PPT.

Since both the beam and target were only partially polarized $\left(P_{\mathrm{B}}\right.$ and $\left.P_{\mathrm{T}}\right)$, the relative cross sections were obtained from the equations

$$
\begin{aligned}
& \frac{\mathrm{d} \sigma}{\mathrm{d} \Omega}(\uparrow \uparrow)=\langle\mathrm{d} \sigma / \mathrm{d} \Omega\rangle\left[1+\frac{4\left(N_{\uparrow \uparrow}-N_{\downarrow \downarrow}\right)}{\left(P_{\mathrm{B}}+P_{\mathrm{T}}\right) \Sigma N_{i j}}+C_{n n}\right], \\
& \frac{\mathrm{d} \sigma}{\mathrm{d} \Omega}(\downarrow \downarrow)=\langle\mathrm{d} \sigma / \mathrm{d} \Omega\rangle\left[1-\frac{4\left(N_{\uparrow \uparrow}-N_{\downarrow \downarrow}\right)}{\left(P_{\mathrm{B}}+P_{\mathrm{T}}\right) \Sigma N_{i j}}+C_{n n}\right], \\
& \frac{\mathrm{d} \sigma}{\mathrm{d} \Omega}(\uparrow \downarrow)=\frac{\mathrm{d} \sigma}{\mathrm{d} \Omega}(\downarrow \uparrow)=\langle\mathrm{d} \sigma / \mathrm{d} \Omega\rangle\left[1-C_{n n}\right],
\end{aligned}
$$

where $C_{n n}$ is given by

$$
C_{n n}=\frac{N_{\uparrow \uparrow}+N_{\downarrow \downarrow}-N_{\uparrow \downarrow}-N_{\downarrow \uparrow}}{P_{\mathrm{B}} P_{\mathrm{T}} \Sigma N_{i j}}
$$




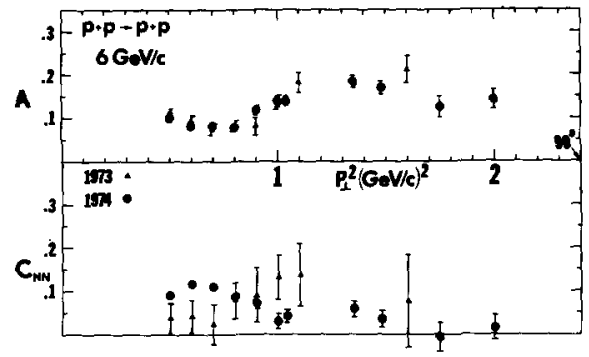

Fig. 2. The asymmetry parameter $A$ and $C_{n n}$ are plotted against $P_{\perp}^{2}$ for pp elastic scattering at $6 \mathrm{GeV} / c$.

and $\langle\mathrm{d} \sigma / \mathrm{d} \Omega\rangle$ is the spin-average cross section. The $N_{i j}$ are the normalized event rates in each spin state. We obtained the asymmetry parameter $\boldsymbol{A}$ by averaging over either the beam or target polarization. This gave the consistency check which typically held within $\pm 1 \%$ :

$$
\begin{aligned}
A & =\frac{N_{\uparrow \uparrow}+N_{\uparrow \downarrow}-N_{\downarrow \uparrow}-N_{\downarrow \uparrow}}{P_{\mathrm{B}} \Sigma N_{i j}} \\
& =\frac{N_{\uparrow \uparrow}+N_{\uparrow \downarrow}-N_{\downarrow \uparrow}-N_{\downarrow \uparrow}}{P_{\mathrm{T}} \Sigma N_{i j}}=\frac{2\left(N_{\uparrow \uparrow}-N_{\downarrow \downarrow}\right)}{\left(P_{\mathrm{B}}+P_{\mathrm{T}}\right) \Sigma N_{i j}} .
\end{aligned}
$$

The values of $A$ and $C_{n n}$ obtained from these cross sections are plotted against $P_{\perp}^{2}$ in fig. 2 along with our earlier data. The agreement is generally good. Notice that $C_{n n}$ has a maximum at around $P_{\perp}^{2}=0.7(\mathrm{GeV})$ $c)^{2}$ which is where $A$ has a minimum and about where the spin average cross section breaks.

The relative cross sections are shown in fig. 3, where we plot for each of the 3 initial spin states ( $\uparrow, \downarrow \downarrow$ and $\uparrow \downarrow=\downarrow \uparrow)$ the ratio of $\mathrm{d} \sigma / \mathrm{d} t$ to $\langle\mathrm{d} \sigma / \mathrm{d} t\rangle$, the normal spin average cross section. The spins are measured perpendicular to the scattering plane. One striking feature is the sharp change in $\sigma_{\uparrow \uparrow}$ and $\sigma_{\downarrow \downarrow}$ at about $P_{\perp}^{2}=0.8(\mathrm{GeV} / c)^{2}$ which is also the position of the first break in the cross section. Another striking feature is that $\sigma_{\uparrow \uparrow}$ is about twice as big as $\sigma_{\downarrow \downarrow}$ for $P_{1}^{2}>1(\mathrm{GeV} / c)^{2}$.

Next we turn to the measurement of the recoil proton spin using the B-polarimeter shown in fig. 1. This observes the scattering of the $225 \pm 30 \mathrm{MeV}$ recoil proton at $P_{\perp}^{2}=0.5(\mathrm{GeV} / c)^{2}$ from a $5^{\prime \prime}$ long carbon block into scintillation counters $100^{\prime \prime}$ away sub. tending the range $\theta_{\text {lab }}=6^{\circ} \rightarrow 12^{\circ}$. By measuring the

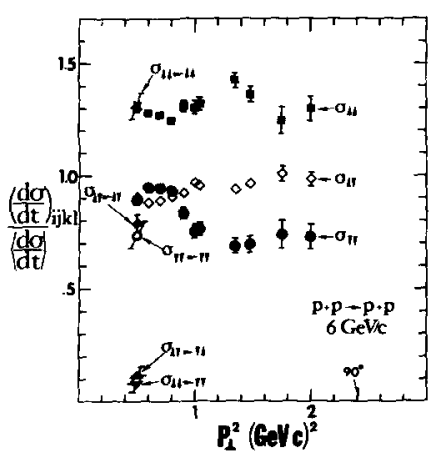

Fig. 3. The ratio of the differential elastic pp cross section for each spin state to the spin average cross section $\langle\mathrm{d} \sigma / \mathrm{d} t\rangle$ is plotted against $P_{I}^{2}$. The spins are measured perpendicular to the scattering plane. The $\sigma_{i j}$ refer to the initial spins only while the $\sigma_{\ddot{j} \rightarrow k l}$ indicate initial and final spins. We observe the scattering of the forward particle to the left as shown in fig. 1 .

asymmetry in p-C elastic scattering to the left (BL) and to the right (BR) we obtain the recoil polarization from the equation

$P_{\mathrm{R}}=\frac{1}{A_{\mathrm{pC}}}\left(\frac{\mathrm{BL}-\mathrm{BR}}{\mathrm{BL}+\mathrm{BR}}\right)$.

We estimated the effective analyzing power to be $A_{\mathrm{pC}}=0.50 \pm 0.05$ using the data of Chamberlain et al. [6] at $289 \mathrm{MeV}$. This assumed that our time of flight measurement removed significant inelastic contamination and that the systematic asymmetry of the polarimeter was $5 \%$ or less.

Thus for each of the 4 initial spin states $(\uparrow \uparrow, \downarrow \downarrow$, $\uparrow \downarrow$ and $\downarrow \uparrow)$ we measure $P_{\mathrm{R}}$ and thus obtain the number of recoil protons in each state

$N_{i j \rightarrow 0 \uparrow}=N_{i j}\left(\frac{1+P_{\mathrm{R}}}{2}\right), \quad N_{i j \rightarrow 0 \downarrow}=N_{i j}\left(\frac{1-P_{\mathrm{R}}}{2}\right)$.

Folding in the partial polarization of the beam and target $\left(P_{\mathrm{B}}\right.$ and $\left.P_{\mathrm{T}}\right)$ we then obtain the cross sections for the 8 different spin states: $\sigma(\uparrow \uparrow \rightarrow 0 \uparrow), \sigma(\uparrow \uparrow \rightarrow 0 \downarrow)$, $\sigma(\downarrow \downarrow \rightarrow 0 \uparrow), \sigma(\downarrow \downarrow \rightarrow 0 \downarrow), \sigma(\uparrow \downarrow \rightarrow 0 \uparrow), \sigma(\uparrow \downarrow \rightarrow 0 \downarrow)$, $\sigma(\downarrow \uparrow \rightarrow 0 \uparrow)$ and $\sigma(\downarrow \uparrow \rightarrow 0 \downarrow)$. Our notation is $\sigma$ (beam, target $\rightarrow$ scattered, recoil) and 0 denotes unmeasured. If we assume parity invariance then all 8 single flip amplitudes must be zero (e.g., $\sigma(\uparrow \uparrow \rightarrow \downarrow \uparrow)=0$ ). Then the 8 cross sections above are in fact the 8 pure spin 


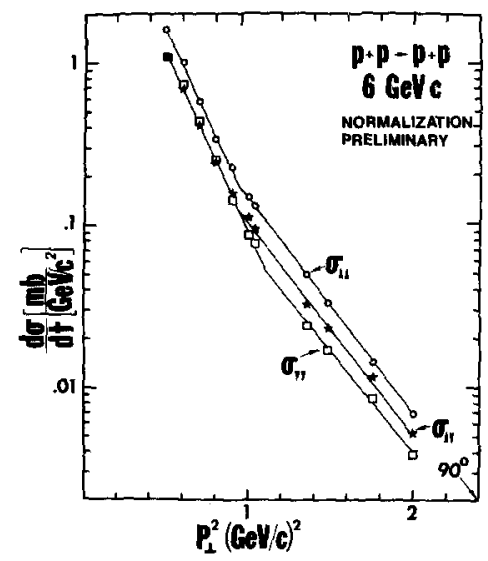

Fig. 4. The differential elastic proton proton cross section for each initial spin state is plotted against $P_{\perp}^{2}$. The spins are measured perpendicular to the scattering plane and the forward particle scatters to the left as shown in fig. 1.

state cross sections: $\sigma(\uparrow \uparrow \rightarrow \uparrow \uparrow), \sigma(\uparrow \uparrow \rightarrow \downarrow \downarrow)$, $\sigma(\downarrow \downarrow \rightarrow \uparrow \uparrow), \sigma(\downarrow \downarrow \rightarrow \downarrow \downarrow), \sigma(\uparrow \downarrow \rightarrow \downarrow \uparrow), \sigma(\uparrow \downarrow \rightarrow \uparrow \downarrow)$, $\sigma(\downarrow \uparrow \rightarrow \downarrow \uparrow)$ and $\sigma(\downarrow \uparrow \rightarrow \uparrow \downarrow)$. Thus we have measured directly the square of the magnitude of the amplitudes for the 8 pure transversity states but have no information about their phases. Rotational invariance of space gives two relations between these

$\sigma(\uparrow \downarrow \rightarrow \uparrow \downarrow)=\sigma(\downarrow \uparrow \rightarrow \downarrow \uparrow)$ antiparallel non flip $\sigma(\uparrow \downarrow \rightarrow \downarrow \uparrow)=\sigma(\downarrow \uparrow \rightarrow \uparrow \downarrow)$ antiparallel double flip

and $T$ invariance give another

$\sigma(\uparrow \uparrow \rightarrow \downarrow \downarrow)=\sigma(\downarrow \downarrow \rightarrow \uparrow \uparrow)$ parallel double flip.

We have assumed that $P$ and $T$ invariance hold and have averaged the corss sections given in eqs. (8) and (9) to obtain the $\sigma(\uparrow \downarrow \rightarrow \uparrow \downarrow), \sigma(\uparrow \downarrow \rightarrow \downarrow \uparrow)$ and $\sigma(\uparrow \uparrow \rightarrow \downarrow \downarrow)$ shown in fig. 3 . Our measurements of the other two of the 5 independent tranversity cross sections $\sigma(\uparrow \uparrow \rightarrow \uparrow \uparrow)$ and $\sigma(\downarrow \downarrow \rightarrow \downarrow \downarrow)$ are also shown.

For the antiparallel non flip we have sufficient precision for a fair test of $P$ invariance. We find that

$\frac{\sigma(\uparrow \downarrow \rightarrow 0 \downarrow)-\sigma(\downarrow \uparrow \rightarrow 0 \uparrow)}{\sigma(\uparrow \downarrow \rightarrow 0 \downarrow)+\sigma(\downarrow \uparrow \rightarrow 0 \uparrow)}=-0.02 \pm 0.05$.

This sets an upper limit on the single flip cross sections $\sigma(\uparrow \downarrow \rightarrow \downarrow \downarrow)$ and $\sigma(\downarrow \uparrow \rightarrow \uparrow \uparrow)$ or more precisely on any differences between these two P-violating cross sections. The other two tests involve smaller double flip amplitudes for which our precision is inadequate.
The most interesting feature seen in fig. 3 is that the double flip cross sections are typically 10 times smaller than the non flip cross sections. The $\sigma(\uparrow \uparrow \rightarrow \downarrow \downarrow)$ cross section is largest being so 7 e $80 \%$ larger than $\sigma(\uparrow \downarrow \rightarrow \uparrow \downarrow)$ and $\sigma(\downarrow \downarrow \rightarrow \downarrow \downarrow)$. Another way to present this data is in terms of the Wolfenstein parameters $D_{n n}$ and $K_{n n}$. We find that $D_{n n}=0.81 \pm 0.10, K_{n n}=$ $0.14 \pm 0.08$.

Finally in fig. 4 we have plotted the differential elastic cross sections themselves against $P_{\perp}^{2}$ rather than the cross section rations. This more clearly demonstrates the relation between the change in the spin dependence and the break in the cross section. However, the normalization of these absolute cross sections is somewhat uncertain because of limited statistics in the radiochemical analysis of the aluminum foils used to calibrate the $\mathrm{N}$ monitor which measures the number of incident beam particles ( $I_{\mathrm{o}}$ in eq. (2)). Fortunately this normalization uncertainty could only shift all the points up or down together by perhaps $20 \%$.

In the "diffraction peak" region of $P_{\perp}^{2}<1(\mathrm{GeV} / c)$ " the three cross sections are parallel and $\sigma(\uparrow \uparrow)$ is about $40 \%$ larger than $\sigma(\downarrow \downarrow)$ and $\sigma(\uparrow \downarrow)$ which are about equal. In the second region after the break $\left[P_{\perp}^{2}>1(\mathrm{GeV} / c)^{2}\right]$ the cross sections are also parallel to each other but $\sigma(\uparrow \uparrow)$ is now about twice as big as $\sigma(\downarrow \downarrow)$; and $\sigma(\uparrow \downarrow)$ is about half-way between them. The sharp breaks and the two cases of rather different but parallel behavior are quite striking.

We are very grateful to the ZGS staff for the successful operation of the polarized beam and to Dr. M. Borghini and Mr. J.A. Bywater for their aid and advice in the construction of PPT V. We thank Ms. S.B. Andrews, Mr. H.E. Haber, and Miss M.M. White for their help in running and Dr. D. Hill for his advice on polarized targets.

\section{References}

[1] P. Grannis et al., Phys. Rev. 148 (1966) 1297.

[2] M. Borghini et al., Phys. Lett. 24B (1967) 77; 31B (1970) 405; $36 B$ (1971) 501;

M.G. Albrow et al., Nucl. Phys. B23 (1970) 445.

[3] N.E. Booth et al., Phys. Rev. Lett. 21 (1968) 651; 23 (1969) 192; 25 (1970) 898; Phys. Rev. D8 (1973) 45. G.W. Abshire et al., Phys. Rev. Lett. 32 (1974) 1261.

[4] E.F. Parker et al., Phys. Rev. Lett. 31 (1973) 783.

[5] J.R. O'Fallon et al., Phys. Rev. Lett. 32 (1974) 77.

[6] O. Chamberlain et al., Phys. Rev. 102 (1956) 1659. 